\title{
Análisis de la caracterización discursiva de los relatos migratorios en Twitter. El caso Aquarius
}

\section{Analysis of the discursive characterization of migratory stories on Twitter: The Aquarius case}

\author{
Ángel Fernández Fernández. Universidad Europea de Madrid. España. \\ angelmiguel.fernandez@ universidadeuropea.es \\ $[\mathrm{CV}](\mathrm{C})$ \\ Almudena Revilla Guijarro. Universidad Europea de Madrid. España. \\ almudena.revilla@universidadeuropea.es \\ $[\mathrm{CV}] \odot \mathrm{C}$
}

Lucía Andaluz Antón. Universidad Europea de Madrid. España.

marialucia.andaluz@universidadeuropea.es

$[\underline{\mathrm{CV}}]$ (1)

Este artículo recoge parte de los resultados del proyecto de investigación "Monitorización y análisis de la caracterización discursiva de los relatos migratorios en Twitter (2018/UEM07)". Se trata de un proyecto de $I+D$ financiado por la Universidad Europea y desarrollado por un grupo multidisciplinar de investigadores pertenecientes a la Facultad de Ciencias Sociales y de la Comunicación y de la Escuela de Arquitectura, Ingeniería y Diseño de la Universidad Europea. El proyecto está vinculado al Grupo de Investigación Comunicación y Migraciones, uno de los grupos de investigación consolidados de la Facultad de CC.SS. y de la Comunicación de la Universidad Europea.

Cómo citar este artículo / Referencia normalizada

Fernández Fernández, Á., Revilla Guijarro, A. y Andaluz Antón, L. (2020). Análisis de la caracterización discursiva de los relatos migratorios en Twitter. El caso Aquarius. Revista Latina de Comunicación Social, (77), 1-18. https://www.doi.org/10.4185/RLCS-2020-1446

\begin{abstract}
RESUMEN
Introducción: La polémica provocada alrededor del rescate y la acogida de los migrantes que viajaban a bordo del Aquarius tuvo un reflejo especialmente importante en Twitter. Objetivos y metodología: El objetivo de este artículo es analizar las representaciones construidas en esta red social alrededor de la migración a partir del estudio del caso Aquarius. Para ello, se han monitorizado durante cuatro meses cerca de dos millones de tuits relacionados con el término Aquarius y se han analizado la influencia de Twitter en la creación y viralización de bulos y la presencia de estrategias discursivas que favorecen el discurso del odio. Conclusiones: Se ha podido observar que en muchos de los tuits publicados se produce una estereotipación de los migrantes, así como la tendencia al predominio de los aspectos emocionales frente a la argumentación racional. Además, se han identificado al menos once bulos relacionados con el desarrollo del caso.
\end{abstract}

PALABRAS CLAVE: Twitter; migración; desinformación; bulos; caracterización discursiva; discurso de odio. 


\begin{abstract}
Introduction: The controversy that emerged around the rescuing and welcoming of the migrants who were on board the Aquarius was prominently reflected on twitter. Objectives and methodology: The objective of this article is to analyze the representations made around migration on this social network based upon the study of the Aquarius case. To do that, we have monitored, for four months, around two million tweets related to the Aquarius term, the influence Twitter has to create and make hoaxes go viral, and the presence of discursive strategies promoting hate speech. Conclusions: We observed that a stereotyping process of migrants occurred in many of the tweets posted, as well as the tendency of emotional aspects prevailing over rational argumentation. Additionally, at least eleven hoaxes related to the coverage of the case were identified.
\end{abstract}

KEYWORDS: Twitter; migration; disinformation; fake news; discursive characterization; hate speech.

\title{
CONTENIDOS
}

1. Introducción. 2. Metodología. 2.1. Análisis automatizado. 2.1.1. Filtrado. 2.1.2. Procesamiento de los tuits. 2.1.3. Análisis de sentimiento. 2.2. Análisis cualitativos. 3. Resultados. 3.1. Los bulos en el caso Aquarius. 3.2. Caracterización de los inmigrantes. 4. Conclusiones. 5. Referencias bibliográficas.

\section{Introducción}

La travesía, y posterior desembarco en Valencia, de los migrantes que viajaban a bordo del Aquarius en junio de 2018 tuvo una amplia cobertura informativa. De hecho, a través de este caso, la inmigración, que tantas veces pasa desapercibida, adquirió una gran relevancia en la actualidad mediática española, ocupando, durante una semana, todo tipo de espacios informativos.

El Aquarius es un barco dependiente de la ONG francesa SOS Méditerraneé, empleado desde 2016 en tareas relacionadas con el salvamento marítimo de migrantes. El barco, que durante los primeros días del mes de junio había rescatado a 629 personas, no pudo atracar en los puertos de Malta e Italia, debido a la negativa de sus gobiernos al desembarco de las personas rescatadas. El 11 de junio de 2018, el Gobierno de España ofreció al Aquarius la posibilidad de que los migrantes, a bordo del barco, desembarcaran en el puerto de Valencia, donde finalmente este atracó el 17 de junio.

Por las diversas variables que concurrían alrededor de este caso, el Aquarius se había convertido en noticia de primera plana, con implicaciones mucho más complejas que las planteadas por la llegada de cualquier otro barco con inmigrantes a las costas españolas. El caso Aquarius favoreció la reflexión pública acerca del tráfico de personas, del papel de los barcos de rescate de las ONG en el Mediterráneo y de la ausencia de una política de inmigración común de la UE, lo que ha llevado a los dirigentes de los países mediterráneos a tomar diferentes decisiones, dependiendo de su ideología o del interés político del momento.

El protagonismo mediático del caso se trasladó también a las redes sociales y muy especialmente a Twitter. Los usuarios intervinieron en el discurso mediático confrontando sus opiniones con el seguimiento informativo de los medios de comunicación, asumiendo el papel de constructores de la opinión pública en torno a la inmigración y expresando discursos racistas y xenófobos, pero también de integración y convivencia. Alesina, Miano y Stantcheva (2018) en su investigación sobre las percepciones erróneas en la inmigración, presentaron en sus conclusiones la idea distorsionada que tienen los usuarios en cuestiones tales como el número y el carácter de los inmigrantes, basada en una imagen absolutamente preconcebida. Estas condiciones propiciaron la difusión y viralización de 
numerosos bulos, que gracias al apoyo de las nuevas herramientas tecnológicas, y a la dinamización de los debates con la ayuda de troles y robots, trasladaron rápidamente mensajes falsos que contribuyeron a la manipulación de la opinión pública (Richter, 2018).

Partiendo del caso Aquarius, esta investigación pretende analizar las características del discurso generado en Twitter alrededor de la migración, determinando de qué manera los mensajes publicados en esta red social colaboran en la creación de juicios sobre las personas migrantes. Se trata por tanto de identificar las características comunes, en el discurso vinculado al relato migratorio, y determinar cómo la realidad de los migrantes que viajaron en el Aquarius se ve deconstruida por los distintos mensajes publicados en Twitter, especialmente por aquellos que favorecen la intoxicación informativa a través de la creación y difusión de bulos, del uso de términos asociados al discurso de odio y de la formación de estereotipos.

El artículo se inscribe dentro de las reflexiones en torno a la construcción del discurso respecto a la inmigración (Van Dijk, 1997; Bañón, 2002) y a la imagen de los inmigrantes en la sociedad española y en los medios de comunicación social (Imbert, 1990; Santamaría, 2002; Retis, 2003; Lafuente, 2010), temas que se han repetido en distintos estudios en las últimas décadas. Los nuevos ámbitos de opinión son las redes sociales y en ellas los usuarios parten de elementos discriminatorios (Acosta, 2015) cuando no de un discurso de carácter populista (Alonso, 2018) en torno a la migración.

\section{Metodología}

La construcción del corpus de este estudio se produce a través de la captura, filtrado y análisis automatizado de los tuits relacionados con el objeto de estudio. Posteriormente, este proceso se complementa con un análisis cualitativo y no automatizado, centrado en los días más relevantes para la evolución del caso.

Con esta finalidad, en este trabajo se han monitorizado todos los tuits publicados con la etiqueta \#Aquarius entre los días 11 de junio y 25 de octubre de 2018. Este periodo, que coincide con el desencadenamiento de la crisis humanitaria generada en torno al rescate y a la acogida de los migrantes que se encontraban a bordo del barco, facilita el análisis de la evolución del sentimiento en los tuits en un contexto suficientemente amplio.

Como ya se ha indicado anteriormente, en una segunda fase del estudio se analizaron de manera independiente los tuits publicados durante los días 11, 12, 13 y 17 de junio. Estos días, que corresponden al período comprendido entre el momento en el que el Gobierno de España decidió acoger a los inmigrantes en el puerto de Valencia y la llegada del barco al mismo, fueron los que concentraron una mayor actividad en la cobertura informativa de este caso.

\subsection{Análisis automatizado}

Los mensajes que integran un primer conjunto de datos, 1.917.041 tuits, se recopilaron directamente a través de la API de Twitter que permite solicitar muestras de tuits. Tras la eliminación de los mensajes duplicados, mayoritariamente retuits, el conjunto original quedó reducido a un total de 273.976 tuits ${ }^{1}$. Partiendo de este dataset se abordó una metodología estandarizada para el análisis de información basada en el filtrado, procesamiento y posterior identificación del sentimiento asociado a cada tuit del corpus resultante.

\footnotetext{
${ }^{1}$ El proceso de filtrado de los tuits duplicados se realizó a través Pandas. Esta librería de Python, creada para la manipulación y el análisis de grandes conjuntos de datos, posee una función que permite eliminar de manera sencilla las entradas duplicadas.
} 


\subsubsection{Filtrado}

El proceso de filtrado comenzó con la detección de los idiomas presentes en el conjunto de datos. Esta tarea se realizó a través de Pyglot, una librería programada en Python que identifica los patrones más reconocibles en las distintas lenguas. Gracias a ella fue posible detectar tuits escritos en 128 idiomas diferentes y, posteriormente, seleccionar los 53.682 que estaban escritos en español.

A continuación, se procedió a la eliminación de los tuits no relacionados con el objeto de estudio y de aquellos elementos carentes de carga semántica. Para ello se crearon tres funciones diferentes. La primera de ellas permitió realizar el preprocesamiento de cada tuit, convirtiendo a minúsculas todos los caracteres incluidos en ellos y eliminando los signos diacríticos y de puntuación.

Posteriormente se crearon dos funciones destinadas a identificar los tuits relacionados con la marca de refrescos Aquarius y con el signo zodiacal y la constelación del mismo nombre. El funcionamiento de ambas funciones se basa en la contextualización del término Aquarius, identificando aquellas secuencias de palabras relacionadas con cada una de estas áreas de significado.

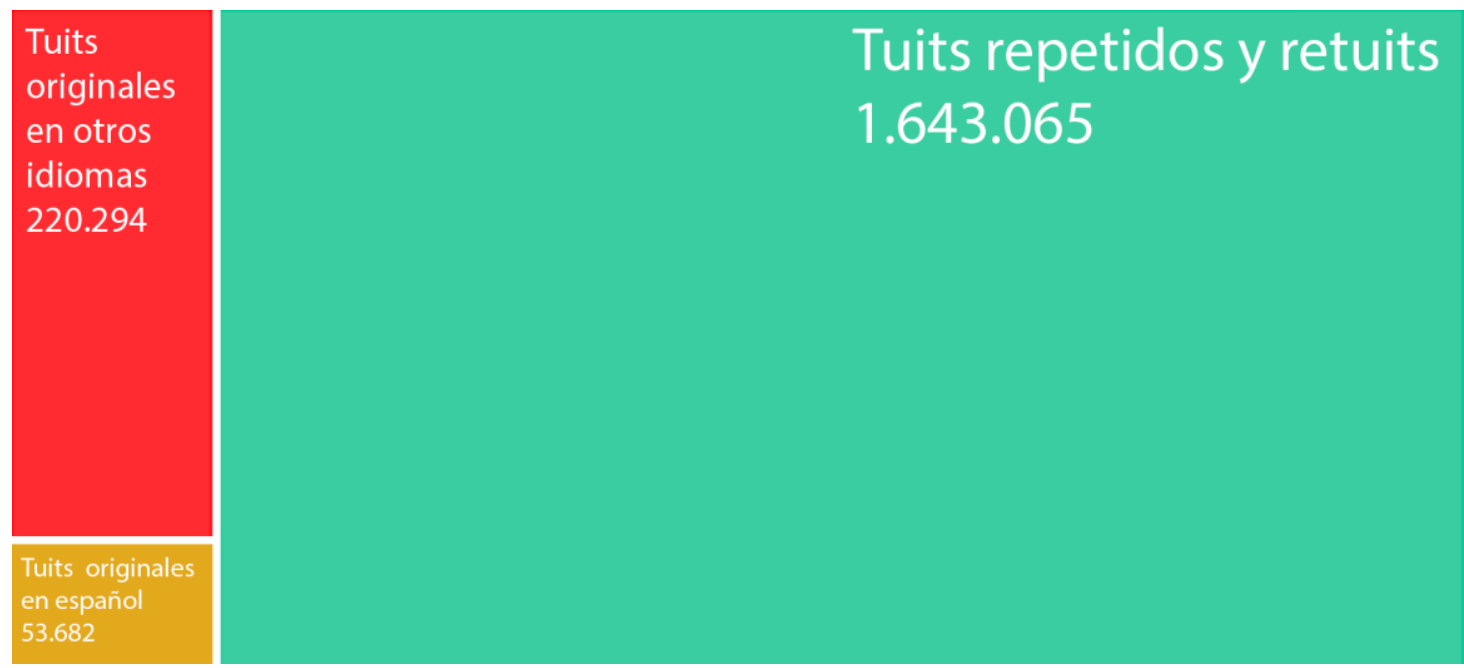

Figura 1: número de tuits repetidos y originales en español y en otros idiomas.

Fuente: elaboración propia.

Finalmente, en una segunda fase de filtrado, se procedió a identificar las palabras vacías o stop words presentes en cada tuit. En el Procesamiento de datos del lenguaje natural (PLN), estas palabras son aquellas que, como los artículos, preposiciones y pronombres, carecen de componente significativa. Por lo general, este tipo de palabras suelen ser eliminadas, sobre todo, si, tal como ocurre en este caso, no influyen en la desambiguación de los términos analizados ${ }^{2}$.

\subsubsection{Procesamiento de los tuits}

El procesamiento de los tuits consistió esencialmente en su lematización y tokenización. La lematización es el proceso lingüístico de obtención de la forma canónica de una palabra o lema a partir de su forma flexionada. A través de él, se pueden transformar palabras en plural o formas verbales conjugadas en el lema correspondiente. Así, mediante la lematización, todos los sustantivos

\footnotetext{
${ }^{2}$ La identificación de palabras vacías se realizó a través de las librerías de Python, NTLK y Stop-words.
} 
que aparecen en plural en nuestro corpus se transformaron a su forma singular, todos los adjetivos a su forma masculina y singular y todas las formas verbales al infinitivo ${ }^{3}$.

En el procesamiento de lenguaje natural, la tokenización consiste en la división de una frase u oración en cada una de las palabras que la componen para facilitar su análisis de forma individualizada. Este proceso puede realizarse con distintas librerías de Python, pero se optó por emplear NTLK con la intención de aportar mayor consistencia procedimental al filtrado.

\subsubsection{Análisis de sentimiento}

Tras las fases de limpieza, traducción y procesamiento del dataset, se procedió al análisis de sentimiento de los tuits que forman nuestro corpus. Para ello, se empleó Vader Sentiment, una herramienta desarrollada por la Python Software Foundation, que realiza un análisis de sentimientos utilizando técnicas de Machine Learning para clasificar y ponderar de forma automática el carácter positivo o negativo del texto en función de las palabras y su contexto.

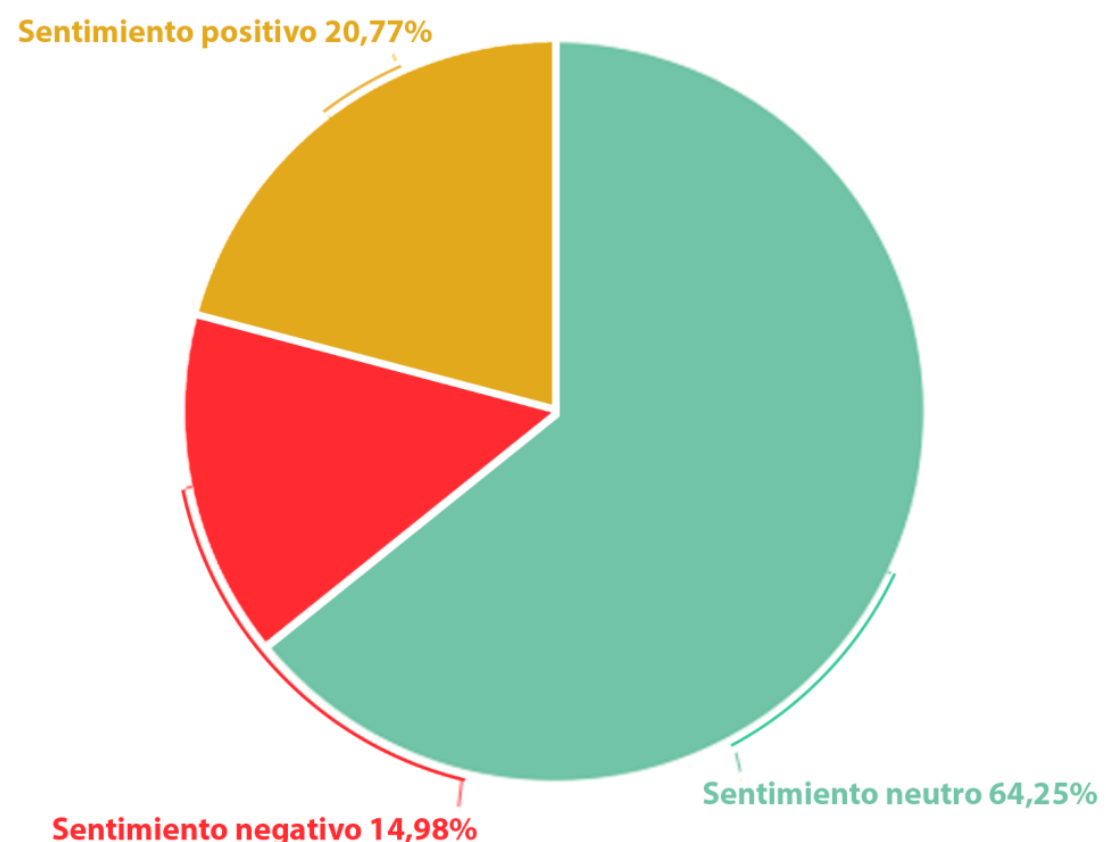

Figura 2: porcentaje de tuits que expresan un sentimiento positivo, negativo y neutro.

Fuente: elaboración propia.

Partiendo de los valores devueltos por esta herramienta se obtuvieron 8.040 tuits con un sentimiento negativo (14,98\%), 11.152 tuits con un sentimiento positivo $(20,77 \%)$ y 34.490 tuits con un sentimiento neutro $(64,25 \%)$.

Si bien este estudio se ha focalizado en el análisis de los tuits que expresan un sentimiento positivo o negativo respecto de los migrantes, se plantea la conveniencia de abordar nuevas investigaciones centradas en el estudio de los mecanismos de inclusión de elementos pragmáticos, contextuales y de la ironía en los procesos automatizados de análisis de sentimiento.

\footnotetext{
${ }^{3}$ La lematización de los tuits que integran nuestro corpus se realizó a través de la herramienta SpaCy.
} 


\subsection{Análisis cualitativos}

Como se ha indicado anteriormente, tras las fases de procesamiento y análisis automatizados, se abordó una fase de análisis cualitativo y no automatizado. Esta se articuló alrededor de dos perspectivas convergentes: por un lado, el análisis de los bulos generados en Twitter alrededor del Caso Aquarius y, por otro, el estudio de la caracterización que se realiza de los migrantes en los tuits que integran nuestro corpus.

Si bien en el análisis de los bulos se optó por estudiar todos los tuits recogidos entre el 11 de junio y el 25 de octubre de 2018, en el análisis de la caracterización se prefirió estudiar los tuits publicados únicamente durante los días 11, 12, 13 y 17 de junio, aquellos en los que se registraron hitos más significativos en la evolución del caso y los que suscitaron una mayor actividad tanto en Twitter como en los medios de comunicación. Como resultado de estas catas, se obtuvieron un total de 23.929 tuits ${ }^{4}$.

Esta diferenciación metodológica se deriva de las características específicas de cada uno de los elementos analizados. Así, mientras que en el análisis de la formación y difusión de bulos resulta fundamental disponer del mayor contexto posible a fin de evidenciar su evolución, en el análisis de la caracterización del discurso resulta mucho más interesante concentrar el estudio en los momentos en los que la conversación en Twitter adquiere mayor intensidad.

Una vez discriminados estos tuits se ha optado por un análisis de contenido en el que se ha observado el tipo de interacción de los usuarios participantes y los elementos lingüísticos, con los que identificar los mensajes, atendiendo a aquellos indicadores comunes dentro de los diferentes argumentos expuestos.

Muchos de los tuits que ocupan nuestro corpus parecen estar descontextualizados al ser comentarios reducidos y sintéticos dependientes de otros argumentos o bien al no tener referencia de otros hashtags sobre el caso que ocupa este trabajo a los que se puede referir fuera del que se ha hecho la monitorización (\#Aquarius), proporcionando algunos resultados incompletos. De ahí que haya sido importante considerar una serie de variables para elaborar el análisis discursivo de los tuits y la construcción de la imagen de las personas inmigrantes dentro del corpus que se ha elaborado.

Primero se ha establecido un tema principal, en este caso, el viaje de los inmigrantes en el barco Aquarius hasta Valencia, no teniendo en cuenta los tuits generados en torno a la decisión política de Pedro Sánchez de traer el Aquarius a España. El ser humano construye su historia y su discurso dando nombres a las cosas, a las personas y a las situaciones. En este trabajo, se ha intentado establecer una taxonomía de las palabras con las que se denomina a las personas del Aquarius, para ser conscientes de las posiciones que ocupan los usuarios de Twitter ante el hecho noticiable y sus protagonistas.

En segundo lugar, se han comprobado las principales temáticas con contenido falso generadas en Twitter alrededor del Aquarius durante la travesía, y se ha tomado una de ellas como referencia para comprobar el tipo de información que difundían, así como la tendencia y la carga emocional del mensaje. También se ha comprobado si ha habido acción de verificación posterior para detectar el bulo, a través de iniciativas y proyectos de fact-checking.

Por último, se ha analizado el contenido de los tuits dirigidos explícitamente a los migrantes a bordo del Aquarius teniendo en cuenta las premisas del discurso de odio y los diferentes tonos: informativo,

\footnotetext{
${ }^{4}$ Del 11 de junio se discriminaron los 10.454 tuits relacionados con el barco Aquarius, el 12 de junio 6.334 , el 13 de junio $3.031 \mathrm{y}$, finalmente, el 17 de junio, día del desembarco en el puerto de Valencia, con 4.110 tuits.
} 
opinativo o puramente emocional, para su discriminación y posterior valoración positiva o negativa respecto a los inmigrantes que llegan desde el mar.

\section{Resultados}

\subsection{Los bulos en el caso Aquarius}

La desinformación, que hace alusión a los bulos y de forma más popularizada pero también más errónea a las fake news, puede definirse como la difusión intencionada de informaciones que mienten en todo o en parte de lo que expresan. Estos contenidos se caracterizan por su falta de rigor y por haber sido creados con la voluntad de manipular a la opinión pública a través de la distorsión de los hechos. De esta forma, consiguen transmitir una realidad ficticia con el objetivo de desestabilizar a la audiencia que los recibe (Olmo \& Romero, 2019).

En esta investigación se han detectado hasta once grandes bulos que han contribuido a crear una imagen negativa y de rechazo hacia los migrantes que viajaban en el Aquarius. El primero que se recoge en la tabla incluida a continuación versa sobre una supuesta declaración realizada por Celia Villalobos, ex diputada del PP: "Lamento que se mueran en el mar, pero en mi país no los quiero ver". Se trata de un bulo recurrente, ya viralizado y desmentido el 21 de marzo de 2017 por iniciativa de Maldita.es, que se puso de nuevo en circulación coincidiendo con el caso Aquarius.

Tabla 1. Los once bulos más viralizados en el caso Aquarius.

\begin{tabular}{|c|c|c|c|}
\hline $\begin{array}{l}\text { Fecha del } \\
\text { desmentido }\end{array}$ & & $\begin{array}{l}\text { Medio que } \\
\text { desmiente }\end{array}$ & Titular del bulo \\
\hline $\begin{array}{l}21 / 03 / 2017- \\
11 / 06 / 2018 \\
12 / 06 / 2018 \\
\end{array}$ & - & Maldito Bulo/ Verne & $\begin{array}{l}\text { Celia Villalobos no dijo "lamento que se mueran en el } \\
\text { mar pero en mi país no los quiero ver" }\end{array}$ \\
\hline $\begin{array}{l}12 / 06 / 2018 \\
11 / 08 / 2018\end{array}$ & - & Verne/Eldiario.es & Los inmigrantes cobrarán una ayuda de 532 euros al mes \\
\hline $12 / 06 / 2018$ & & Verne/ ONGD & $\begin{array}{l}\text { Los inmigrantes vienen a aprovecharse de los servicios } \\
\text { sociales }\end{array}$ \\
\hline $12 / 06 / 2018$ & & Verne & Otros 729 inmigrantes más se suman al Aquarius \\
\hline $12 / 06 / 2018$ & & Verne & ¿Y si llegan terroristas? \\
\hline $18 / 06 / 2018$ & & Maldito Bulo & $\begin{array}{l}\text { No, esta imagen de inmigrantes con móviles y tabaco no } \\
\text { se corresponde con los inmigrantes del Aquarius }\end{array}$ \\
\hline $\begin{array}{l}19 / 06 / 2018- \\
21 / 06 / 2018- \\
26 / 06 / 2018 \\
\end{array}$ & & $\begin{array}{l}\text { Diario.es/ Maldito } \\
\text { Bulo/ Distrito.es/ } \\
\text { Vertele/ Ecoteuve }\end{array}$ & $\begin{array}{l}\text { No, Bertín Osborne no ha dicho: "¿Por qué no van todos } \\
\text { los inmigrantes a la mansión de Pablo Iglesias?" }\end{array}$ \\
\hline $25 / 06 / 2018$ & & Maldito Bulo & $\begin{array}{l}\text { No ha habido violaciones a bordo del Aquarius: ni Oltra } \\
\text { lo ha dicho ni hay pruebas }\end{array}$ \\
\hline $\begin{array}{l}25 / 06 / 2018 \\
08 / 11 / 2019\end{array}$ & - & $\begin{array}{l}\text { Maldito Bulo } \\
\text { /Eldiario.es /Newtral }\end{array}$ & $\begin{array}{l}\text { No, la ropa en los contenedores no ha sido tirada por los } \\
\text { inmigrantes del Aquarius }\end{array}$ \\
\hline 25/06/2018 & & Maldito Bulo & $\begin{array}{l}\text { No han desahuciado a una mujer mayor de su casa para } \\
\text { acoger refugiados }\end{array}$ \\
\hline 26/06/2018 & & Maldito Bulo & $\begin{array}{l}\text { No, la CIA no ha avisado al Gobierno de que entre los } \\
\text { migrantes del Aquarius hay soldados de Boko Haram }\end{array}$ \\
\hline $14 / 08 / 2018$ & & Maldito Bulo & $\begin{array}{l}\text { No, Ada Colau no ha ofrecido contratos de trabajo a } \\
\text { todos los inmigrantes del Aquarius }\end{array}$ \\
\hline
\end{tabular}

Fuente: extraído de «Las mentiras que se repiten sobre los migrantes a bordo del 'Aquarius'», 2018,

(C) EDICIONES EL PAÍS S.L; «Los bulos sobre los refugiados y migrantes del Aquarius», 2018, maldita.es - Contenido bajo licencia Creative Commons BY-SA. 
Otro bulo destacable, y señalado en la tabla, es el que plantea la existencia de un supuesto mensaje de la CIA al gobierno español alertando acerca de la presencia de terroristas de Boko Haram entre los migrantes del Aquarius. Se trata de un meme que adquirió relativa relevancia al ser interpretado como un mensaje de carácter oficial. Tras una búsqueda del término "Boko Haram" en el universo de tuits que forman el corpus, se encontraron un total de 18 tuits, de los cuales 14 ayudaron a difundir el bulo de forma directa. Para detectar otros mensajes similares sobre este mismo bulo se filtró el término "terrorista", obteniendo un total de 44 tuits, 17 de los cuales fueron difusores directos del mismo.

También se han identificado tuits con un evidente carácter partidista: "Solo una quinta parte de los emigrantes de ese barco provienen de la guerra, el resto son mafias organizadas de más de 20 países, sin contar posibles terroristas entre los mismos https://t.co/WyIkOY4bdd " (12/06/2018). El enlace incluido en este tuit redirige al usuario a la cuenta oficial de VOX en Twitter, donde aparece una imagen con el siguiente mensaje: “? En VOX nos preguntamos a quién beneficia el tráfico de personas, aprovechándose de su desesperación, con casos como el de \#Aquarius. Los españoles pagaremos cara la irresponsabilidad de Pedro Sánchez y su actitud colaboracionista con las mafias de la inmigración ilegal" (12/06/2018).

Dicho tuit, además de narrar un hecho que no se ajusta a la realidad, nos redirige a una cuenta que alberga contenido político de partido, y que, además, fomenta la indignación respecto a la llegada de migrantes a España. Así, es fácil distinguir en estos mensajes algunas de las emociones más habituales en las redes sociales, tales como el miedo, la furia o la competitividad. Emociones que generan estados de rabia e indignación en el individuo, y que se pueden magnificar y manipular para obtener un beneficio concreto: "La ficción es más lucrativa que las noticias reales, porque genera emociones. Las fake news están diseñadas para indignar” (Peirano, 2019, p. 48).

Al analizar las respuestas generadas en cadena a partir de estos bulos, se diferencia fácilmente la forma de pensar del usuario que interacciona. Este tiende a identificarse con el mensaje, y a su vez con esta comunidad social en lo que ha venido a denominarse como "emociones grupales", tomemos como ejemplo el siguiente tuit: "Comprendo los problemas humanitarios. Conozco españoles que apenas llegan a fin d mes, pensionistas que malviven, jóvenes sin expectativas.... Muchos vienen en busca de oportunidades laborales, pero también hay guerrilleros $\mathrm{q}$ han asesinado, terroristas infiltrados... \#Aquarius" (11/06/2018). Estos usuarios piensan y comparten la orientación emocional del grupo con el que se identifican (Bar-Tal, Chernyak-Hai, Schori \& Gundar, 2009).

Twitter, a diferencia de otras redes sociales es un circuito abierto que favorece el debate. En él, la mayor carga emocional no suele estar en el primer mensaje, sino que aumenta a medida que la conversación se desarrolla. No obstante, puede considerarse como elemento positivo de esta red, a diferencia de WhatsApp, por citar un ejemplo, la posibilidad de desmentir un bulo de manera rápida, permitiendo que los medios de comunicación activen los mecanismos que tengan a su alcance para detener la proliferación de informaciones falsas. Las nuevas iniciativas de verificación periodística o fact-checking que surgieron a principios del siglo XXI a nivel internacional son un buen ejemplo de cómo combatir los bulos en los medios sociales y fuera de ellos.

A diferencia de la verificación "tradicional" que los periodistas realizan antes de publicar una notica, la función principal de la verificación periodística basada en las técnicas de fact-checking es comprobar la información después de haber sido publicada. Como señala Bill Adair, fundador de Politifact: "Verification is the editorial technique used by journalists -including fact-checkers- to verify the accuracy of a statement" (Verification Handbook, 2013). Esta verificación consistiría en comprobar si las declaraciones realizadas por alguien, especialmente responsables políticos, o los 
contenidos generados por los usuarios, son verdaderos, falsos o imprecisos, a través de la comprobación de datos y hechos.

Estas iniciativas actúan como cortafuegos de la desinformación en los medios sociales. Los bulos que se viralizaron durante la travesía del Aquarius fueron desmentidos en primer lugar por el diario El País, a través de un tuit publicado en @el_pais, cuenta oficial de este medio en Twitter. En él se enlazaba a un artículo publicado el 13 de junio de 2018 en su sección Verne en el que recogían los bulos que circulaban sobre el caso Aquarius. En su caso, desde @ElObjetivoLaSexta se publicó el 17 de junio un tuit con un fragmento en vídeo de su programa en televisión protagonizado por Clara Jiménez, fundadora de Maldita.es, desmintiendo los bulos más populares: "Con la llegada del \#Aquarius han vuelto a salir en las redes los bulos sobre los refugiados... @cjimenezcruz los repasa...”. De la misma forma, @Malditobulo también hizo su propia publicación para desmentir el tuit el 26 de junio de 2018 que generó 26 comentarios, 495 retuits y 284 me gustas, con el mensaje: "No. La CIA no ha avisado al Gobierno de que entre las personas que han llegado en el Aquarius haya soldados de 'Boko Haram' Nos lo confirma el Ministerio del Interior. No hay ninguna prueba de lo contrario. La frase de Trump tampoco es real".

Acompañando al tuit la siguiente imagen:

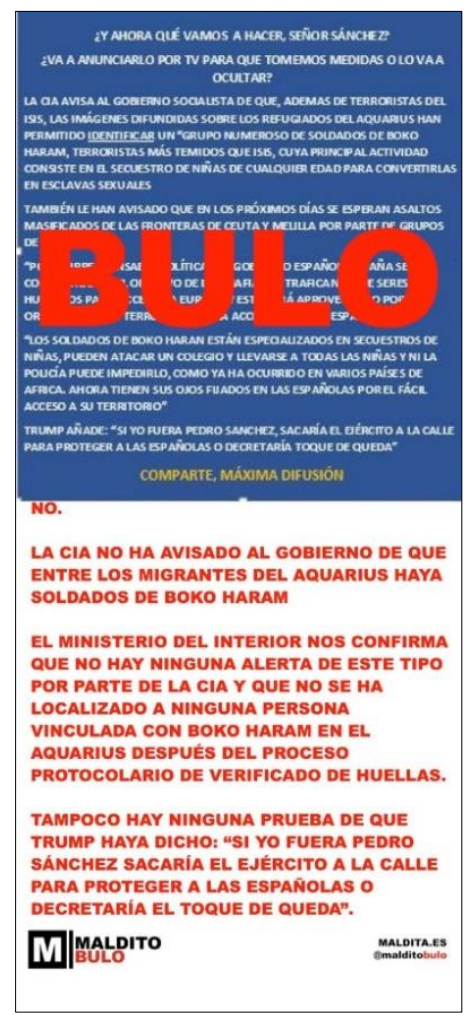

Figura 3: Meme y desmentido del bulo que se viralizó con el mensaje "La CIA ha avisado al Gobierno de que entre los migrantes del Aquarius hay soldados de Boko Haram”de@Malditobulo maldita.es - Contenido bajo licencia Creative Commons BY-SA.

\subsection{Caracterización de los inmigrantes en el caso Aquarius}

Los medios tradicionales han sabido frenar el abuso de términos discriminatorios, con la redacción de guías y libros de estilo, y así elaborar un discurso más respetuoso con los migrantes. Sin embargo, 
en las redes sociales es muy difícil poder acotar los contenidos de los mensajes sin una intervención directa de las cuentas de los usuarios y fomentar un discurso más respetuoso respecto a determinados asuntos en "this defining moment in cyber hate's history" (Citron \& Norton, 2011, p. 1.435).

En el corpus de este trabajo, extraído de Twitter, se han encontrado interacciones y comentarios de los usuarios cargados de violencia contra la inmigración y contra las personas que llegaban en el Aquarius a Valencia. Unos textos con emotividad catártica y descalificaciones simplistas (Trejo Delarbre, 2017) que se podrían incluir dentro del denominado hate speech (discurso de odio): "La forma de expresión, que difunda, incite o justifique el odio racial, la xenofobia, el antisemitismo u otras formas de odio basadas en la intolerancia" (Comisión Europea, 1997).

Una definición bastante amplia que el Código penal español en el artículo 510 acota de la siguiente manera: "[Recibirán castigo] quienes públicamente fomenten, promuevan o inciten directa o indirectamente al odio, hostilidad, discriminación o violencia contra un grupo, una parte del mismo o contra una persona determinada por razón de su pertenencia a aquél, por motivos racistas, antisemitas u otros referentes a la ideología, religión o creencias, situación familiar, la pertenencia de sus miembros a una etnia, raza o nación, su origen nacional, su sexo, orientación o identidad sexual, por razones de género, enfermedad o discapacidad" (2019).

Sin embargo, aunque es una evidencia que el discurso de odio irrumpe en las opiniones de los tuits entorno a los temas de inmigración y, en este caso del Aquarius, no es una excepción, son mucho más numerosas las expresiones hostiles y de violencia las que intercambian desde el plano puramente ideológico y de partidos ante la decisión del Gobierno de España de que el barco atraque en el puerto de Valencia ${ }^{5}$. Pero no por ello, en los comentarios en torno a la inmigración y a las personas inmigrantes del Aquarius, en los que se ha centrado el estudio, se construye un discurso más positivo, sino que, por el contrario, se tergiversa la imagen de las personas rescatadas por la embarcación en una actitud maniquea estableciendo dos bloques diferenciados. En ambos hay una clara reafirmación de pertenencia de grupo de los usuarios: en pro o en contra de la política migratoria llevada a cabo y, en consecuencia, de una polarización entre ellos (los que vienen de fuera) y nosotros (los que vivimos en España).

De nuevo una construcción identitaria de los inmigrantes estereotipada y con tipificaciones negativas (Revilla, 2011) a la que conduce dicha dualidad, dando pie a "sistemas sociales de dominación y desigualdad" (Van Dijk, 2011, p. 294) como los que se producen con los inmigrantes.

"Elegir un nombre u otro, añadir un adjetivo o suprimirlo, favorece o dificulta una determinada representación de la realidad" (Portolés, 1997, p. 21) y la presentación homogénea de los migrantes, desde el nosotros, como construcción de una imagen positiva, y ellos como imagen negativa, determina muchas de las estrategias y mecanismos lingüísticos que se asocian a los inmigrantes (De la Fuente, 2006).

Los nombres con los que se les asigna y referencia son adjetivos sustantivados: "inmigrantes", con una carga semántica más allá de su propio significado de persona que llega de un país no

\footnotetext{
${ }^{5}$ Cabe destacar que en muchos de los tuits tanto de los usuarios que apoyaban la política migratoria establecida por el gobierno de España como de los que disentían de dicha política se recogieron mensajes difamatorios e intimidatorios. Algunos ejemplos: "Eres de una ruindad asquerosa. ¡Ojalá nunca tengas que verte en la piel de los del \#Aquarius! ¡Ah, no, espera, que tú levantas 8000 €uritos al mes de un Estado del que reniegas! Valiente hijo de mala sangre. Además de "sonreír" ya vemos tu catadura moral. https://t.co/vDhhNfswWU” (respuesta a un tuit de Gabriel Rufián); "Si te atreves a plantear la posibilidad de abandonar en alta mar a 600 personas es que eres un Hijo de Puta muy grande! \#Aquarius"; "Diría que tenemos el país en manos de mongolos y traidores, pero es que ya no tenemos país. \#Aquarius".
} 
desarrollado; "africanos", término con proveniencia geográfica en los tuits en positivo, pero también en otros mensajes con cierta carga despectiva, y "refugiados". Este término se utiliza de forma irónica (las comillas le dan ese valor de cambio de sentido en el texto del comentario), cuestionando lo que significa serlo y las dificultades que entraña: “¡Eh,! Que yo me he caído del \#Aquarius, pero iba en el barco también. ¿por dónde puedo pasar a recoger mi pack? Los "refugiados" del Aquarius tendrán empleo remunerado, vivienda y libre circulación en España https://t.co/YCsatfVbnf $(13 / 06 / 18)$.

Este comentario toma el texto prácticamente literal del titular de una noticia publicada por un medio de comunicación de internet, OKdiario, que amplía la información, y clarifica a qué se refiere con los beneficios citados, en el cuerpo de la crónica. Los medios son responsables de trasladar la información, pero los usuarios de Twitter también lo son de manipularla o extraer los términos que benefician su postura en el comentario emitido. La opinión dependiente de las referencias noticiosas en torno al caso se mezcla con una parte anecdótica de la información y otros componentes como las creencias preestablecidas de los usuarios. Así, se presenta como dato cierto aquello que puede ser más producto del interés, del deseo o de una mala interpretación.

Los migrantes del Aquarius, "víctimas" de una situación político-social, son compadecidas también por muchos de los usuarios que intentan empatizar con esas personas y comprender su situación de vulnerabilidad: "A mí sí me enorgullece que ante una crisis humanitaria mi país acoja a las víctimas del \#Aquarius y no se trata de poner tu casa porque hay mecanismos y recursos que funcionan a la perfección" (11/06/18). Olvidando que estas personas no buscan la compasión si no el poder ser sujetos con derechos y responsabilidades en el país de acogida (Gallardo, 2008).

Algunos internautas muestran su orgullo ante una reacción a favor de los inmigrantes, aunque no la definitiva solución, dependiente de políticas eficaces, seguras y humanas ("Antes también, pero desde q soy madre el sufrimiento de un niño me duele horrores. Viendo esos pequeños con una mínima oportunidad de vivir mejor de lo q podían en su país...estoy emocionada, 17/06/18; "\#Aquarius, una solución necesaria para 629 víctimas de la gestión del primer mundo. Ahora hay que buscar una solución para que no haya más Aquarius", 12/06/18), para que dejen de ser "náufragos", personas desesperadas perdidas y sin destino ("Y cómo se llaman aquellos que se encuentran en un barco a la deriva en medio del mar? Náufragos ¿no? Y cuál es la legislación internacional de asistencia a \#naufragos? Pues ya está. Queda dicho. Solo queda prestar socorro y dejarse de excusas, 11/06/18).

O la denominación de "esclavos", palabra que aparece como contraposición de "mafia" o "traficantes", términos con los que identifican a las ONG o a la tripulación del Aquarius. Sin olvidar que pueden seguir siendo esclavizadas las personas que llegan en el barco cuando tomen tierra en España: "Cuando lleguen aquí serán tratados como esclavos siendo mano de obra barata" (12/06/18).

"Inmigración ilegal”, "inmigración irregular", son sintagmas ya de por sí con un alto contenido de significado que, en muchas ocasiones, generan un "poder simbólico" (Bourdieau, 1977). Etiquetas que provienen de la inacción social y de unas insuficientes políticas de inmigración que convierten a los inmigrantes en infrasujetos, con limitaciones de sus derechos. Además, si se acompaña a estos términos con adjetivos como "masiva", contribuyen a identificar la situación del Aquarius con un riesgo para la sociedad y a recalcar cómo los migrantes son sujetos que se encuentran fuera de la norma establecida: "Y con el caso \#Aquarius queda oficialmente abierta la temporada de masiva inmigración ilegal a acoger en nuestro país Que más da el 15\% de paro, $40 \%$ paro juvenil, deficit en administraciones, servicios públicos colapsados, que vengan 600 más https://t.co/DzUrybcNYt" $(11 / 06 / 18)$. 
Las metáforas "invasión” (“¡Viva \#Italia y la \#LigaNorte! Gente seria frente a la \#invasion de la inmigración ilegal", 13/06/18), "avalancha" ("Es de necios y demagogos negar la avalancha de pateras con el efecto llamada de la acogida del \#Aquarius", 17/06/18) y "oleada"6 ("¿Que va a pasar cuando en Africa se corra la voz de que el PM de España te recibe personalmente cómo refugiado? Llegarán oleadas de barcos con africanos", 11/06/18) reafirman lo dicho: movimientos impetuosos de mucha gente y con intención amenazante.

Estos términos resumen dos de las posturas de los usuarios contrarios a la llegada del Aquarius a Valencia: la inmigración como problema gravoso y sin ley que se reduce al establecimiento de un estado de conflicto y delincuencia, que más adelante se comentará.

En este artículo se han categorizado también las expresiones y los términos que fomentan el odio dentro del discurso de los tuits de nuestro corpus de una manera más o menos explícita, o más o menos simbólica, más dependiente del contexto, y para ello se ha hecho una división básica.

Con la invectiva se establece una desvalorización de los inmigrantes, denigrante y despectiva. El uso en el discurso de términos que insultan, que agreden, y que no requieren de otro tipo de contexto para saber que estamos ante una agresión explícita:

"\#aquarius Me cago en los moros! Me cago en todos sus defensores! Me cago en este gobierno traidor!" (11/06/18). O la creación de términos despectivos ("moronegrada") a los que se suma la metáfora de la "ola", un movimiento que será difícil de controlar, y que termina con una clara amenaza de "expansión": "Tal y como llegue la moronegrada, la ola de "xenofobia e insolidaridad" va a llegar a España. Algunos nos vamos a encargar de expandirla https://t.co/kSQ1N8AArN" $(11 / 06 / 18)$

También hay en casos que se utiliza un tono irónico, con el que minimizar esa carga semántica. Sin embargo, lo que hace es reafirmar los prejuicios existentes hacia una raza o un color de piel: "Yo no soy racista, anda que no animo a los futbolistas negros de mi equipo. \#Aquarius" (11/06/18) o "Éramos pocos y parió la abuela... Que ya nos sobran DEMASIADOS MORITOS BUENOS, leches!!!!” (11/06/18). En este último caso, además, del intensificador "demasiados", la fuerza del diminutivo reproduce el contexto pragmático en el que está inmerso y contrapone el valor afectivo del diminutivo a la desvalorización, incorporando desdén y desprecio. Además, el uso del adjetivo "buenos", conduce hacia un significado irónico.

En los tuits no se hallan los porqués ni las causas que han producido el hecho noticioso, ni se da pie en los comentarios a posibles soluciones, sino que hay una ampliación de los tópicos, una competencia por llamar la atención de los usuarios de Twitter con vulgarismos que imprimen una mayor agresividad incurriendo en la acusación de un delito: "600 pollas invasoras más buscando dónde meterla, y el país en déficit hídrico. Cuándo tendremos suficiente saturación demográfica y tercermundismo para estar contentos?" (11/06/18).

Este lenguaje soez con acusaciones e insultos se usa de modo directo bajo el amparo del anonimato y de la virtualidad de la interacción. Los tuits reproducen un discurso mínimo, repetitivo, donde utilizan la figura retórica de la simplificatio, con la que estigmatizan a los inmigrantes, señalándoles como una amenaza social, como generadores de conflictos: "Que tranquilidad me proporciona acoger a violadores y delincuentes en mi tierra. No tenéis vergüenza \#VikingosTeam https://t.co/epWjv0vVTv"

\footnotetext{
${ }^{6}$ Dentro de los 23.929 tuits que integran el corpus, el término avalancha aparece 11 veces. Las palabras invasión y oleada están presentes respectivamente en 20 y en 5 tuits.
} 
(13/06/18); "Y no es un caso aislado. Pasa a diario porque no se controla la inmigración y entran delincuentes en tropel. ¿Los perjudicados? Los españoles y los inmigrantes que vienen a trabajar de verdad en busca de una vida mejor pero claro, impera el buenismo descerebrado. https://t.co/XJtO4kPehW" (17/06/18).

"Tropel", "demasiados", “desbordados", cuantifican al alza y definen situaciones de racismo, de xenofobia o, en todo caso, de aporofobia, miedo y rechazo hacia la pobreza. "Es la fobia hacia el pobre la que lleva a rechazar a las personas, a las razas y a aquellas etnias que habitualmente no tienen recursos y, por lo tanto, no pueden ofrecer nada, o parece que no pueden hacerlo" (Cortina, 2017, p. 21). Estos, y otros comentarios referidos al caso Aquarius, aunque parezcan estar construidos desde una retórica de superioridad, delatan que el usuario que los escribe siente su identidad cultural y nacional amenazada frente a la del inmigrante (Cea D'Ancona, 2009) y por ello rechaza aquellos tratos favorables que pudieran tener las personas que llegan de fuera de España por parte de las instituciones del país. Como ejemplo, algunos tuits extraídos del corpus sobre las supuestas ayudas que reciben los inmigrantes: "El típico excremento humano q prefiere traer gente de fuera para darles casa, paga, ayudas, etc. mientras tiene a compatriotas en parques durmiendo, familias sin trabajo y nuestros mayores sin pensiones dignas" (13/06/18); "Los que vienen a Valencia este viernes, serán acogidos como refugiados y cobraran una paga de 532 euros al mes, ya tienen casas esperándoles y todas las necesidades cubiertas. Esos 629 nos costara: 532X629X12=4.015.536"; "Una paga de 500€ por cabeza que va a salir de nuestros impuestos. Las mujeres ya podéis andar con cuidado que de ese barco van a bajar varios violadores" (17/06/18).

Estos discursos establecen unas comparaciones discriminatorias e imposibles sin el conocimiento de otras variables: "Primero arreglar todos esos españoles sin trabajo o jubilados que tienen que ir a contenedores de basura para comer porque no les llega... y despues los demas.. como sigais asi no os voto ni de coña... arreglar primero este país" (11/06/18); "OJO //"629 INMIGRANTES A EXPAÑA. Manutención, alojamiento, ayudas económicas, escolarización, sanidad, vestimenta... Cada uno de ellos nos costará más de 2.000 euros al mes como poco. 1,4 millones al mes. 17 millones de euros al año. Mientras tanto los españoles" (11/06/18).

Con estos comentarios se inocula el miedo: todo un colectivo busca arrebatar los derechos a los ciudadanos del país que les acoge: "El \#Aquarius se dirige a España. Si peligran las pensiones es fácil, quitamos las ayudas a la inmigración ilegal promovida por las mafias y voilá, ahorro!" $(13 / 06 / 18)$.

Finalmente, a los migrantes se les incapacita para integrarse en la sociedad a la que llegan con una supuesta explotación del sistema asistencial y aprovechamiento del estado del bienestar, situándoles en una situación de déficit social y económico: "Pero cuando trabajan estos infelices? Son contratados como violadores profesionales, manteros o creadores de disturbios? Porque para eso se pulen bastante https://t.co/ffCd0alwWD” (17/06/18).

El grado de agresividad verbal va in crescendo paralelamente a la identificación con una afiliación de grupo que repite en sus comentarios el rechazo hacia los inmigrantes. Un tono de marcado carácter ideológico que justifica una hipotética tendencia delictiva de los inmigrantes: violadores, asesinos... "Sabemos quién son? Sabemos lo que han hecho? Sabemos si son delincuentes O entre ellos hay algún asesino? No sabemos a quien van a meter en España. Más gasto y más inseguridad" $(11 / 06 / 18)$.

E incluso terroristas: "Pues mala suerte para ellos, pobres. El y cia han decidido que los recursos para los refugiados, entre los que van violadores, no sabemos si hay yihadistas" (13/06/18). 
No se ha podido establecer si las falacias de las que se hacen eco los usuarios de Twitter son por ignorancia, ante un pretendido conocimiento, o se utilizan con el propósito de desinformar y crear un clima de distorsión de lo real que lleva a generalizaciones excesivas y a una serie de atributos negativos del inmigrante. Pero, según lo expuesto en este trabajo, muchos de los comentarios, se basan en bulos, en noticias o hechos falsos o malinterpretados. Algunos se achacan a personajes públicos con relevancia, como Mónica Oltra ${ }^{7}$, vicepresidenta de la Generalitat Valenciana: "Hay que atender a las mujeres que han podido sufrir agresiones sexuales durante la travesía". ¿Está diciendo abiertamente que hay violadores en el barco que viene hacia Valencia? \#Aquarius" (13/06/18), o a instituciones consolidadas, como la Guardia Civil: "Guardias civiles alertan ante la llegada del \#Aquarius: "España no puede más" (17/06/18). De esa manera, no se asumen responsabilidades directas sobre lo que se ha escrito o buscan un apoyo en lo mediático para dar mayor credibilidad a sus comentarios.

Además, algunos de esos tuits, que se repiten a lo largo de los días, utilizan deliberadamente un tono provocador, de ruptura con el hilo principal de Twitter que revela su posible condición de troles: “Por qué llaman catástrofe humanitaria lo que es una INVASIÓN a Europa? (11/06/18); “\#Aquarius -un moderno traficante de esclavos- busca puerto para descargar a los 629 inmigrantes que ha recogido haciendo más eficaz el trabajo de las mafias de tráfico de esclavos" (11/06/18).

En definitiva, entre los tuits analizados en este trabajo, se halla un discurso enfatizador y homogéneo de la figura del inmigrante que obstaculiza un mayor conocimiento de las personas que se encontraban en el Aquarius, salvo aquellos tuits que pertenecen a los medios que realizaron un seguimiento informativo, y que los presentaban de forma más individualizada, dándoles nombre y rostro. Los periodistas avisaban en los tuits de sus trabajos informativos sobre las condiciones en las que llegaban desde África y algunas historias de superación: "Hay más de una decena de mujeres embarazadas y de un centenar de menores. Otros tantos, con heridas en la piel por culpa del combustible y del agua del mar" (12/06/18).

El análisis se ha centrado en las denominaciones que han recibido los inmigrantes en los comentarios recogidos en el corpus y el tratamiento negativo y estereotipado, si bien es cierto, que la gran mayoría de los tuits se han redactado en defensa de los inmigrantes, más allá de la toma de decisión del gobierno de España: "Ponerles cara a los migrantes del \#Aquarius ayuda mucho a que algunos retrasados entiendan que son personas. No son 629 números que quieren robar trabajos, son 629 personas $(11 / 06 / 18)$.

Este trabajo evidencia que aún unos pocos utilizan a los inmigrantes como cabeza de turco para atribuirles la culpa de los problemas o decisiones políticas de nuestra sociedad y, aunque se intente a través de hechos y datos comprobados corregir al individuo que tiene una falsa creencia, este no solo tiende a mantener su opinión, sino que, además, se aferra con más fervor a sus ideas (Mantzarlis, 2017). Únicamente un auditorio bien informado será quien rechace los bulos y los argumentos basados en la exaltación y en las emociones más bajas frente a la argumentación racional que dé origen a una reflexión sobre la situación de la inmigración en Europa.

\footnotetext{
7 Mónica Oltra, en una entrevista concedida al canal Cuatro (Programa Cuatro al Día, 13 de junio), hizo unas declaraciones poco afortunadas al decir que se requeriría "atención especializada [para] mujeres que hayan podido tener algún tipo de agresión sexual, violación o incluso sean víctimas de trata durante la travesía”. Según la vicepresidenta, se malinterpretaron sus declaraciones: se estaba refiriendo a violaciones durante la travesía desde los países de origen, antes de ser rescatados por Médicos Sin Fronteras en el Aquarius. Así, recogió este bulo de las supuestas violaciones Maldita.es: https://maldita.es/malditobulo/no-ha-habido-violaciones-abordo-del-aquarius-ni-oltra-lo-ha-dicho-ni-haypruebas/
} 


\section{Conclusiones}

El resultado que arroja el análisis de sentimiento automatizado de nuestro corpus evidencia que, a pesar de la polémica suscitada en torno al caso Aquarius y de su gran repercusión en los medios de comunicación, la mayor parte de los tuits publicados no plantean un sentimiento ni positivo ni negativo sobre el mismo.

No obstante, un análisis más profundo de los tuits evidencia que los usuarios tienden a anteponer sus creencias ideológicas y políticas frente a la "información factual" que revela una realidad opuesta a la creencia que se tiene. Esto quiere decir que, si bien la difusión de una realidad, tal y como ha sucedido, debería ser suficiente para tomarla como verdadera, las orientaciones ideológicas y las "emociones grupales" generadas en las personas, siguen siendo más firmes a nivel general que la propia realidad.

Si bien existen bulos cuyo objetivo es dañar la imagen o la reputación de otros individuos, en la mayoría de los casos lo que se pretende es obtener algún tipo de beneficio. Para combatir la desinformación y evitar la manipulación de la opinión pública basada en las emociones y no en los datos y en los hechos, se hace imprescindible que los medios de comunicación trabajen de forma conjunta.

En este caso, se ha comprobado que, si bien el trabajo de fact-checking se ha hecho de forma correcta, no son muchos los medios de comunicación que se han implicado en esta tarea. Aunque existen más iniciativas inmersas en esta labor de verificación, lo cierto es que, hasta el momento, esta disciplina no tiene un lugar relevante en las redacciones de medios en España, algo que resulta sin duda preocupante dado el volumen de contenidos de dudosa procedencia que llegan diariamente a las redacciones.

Asimismo, este análisis permite comprobar cómo se refuerza la estereotipación en torno a las personas migrantes por la escasa presencia de información y de fuentes contrastadas, como sí ocurriría en los medios de comunicación. Los discursos se reducen a opiniones y a una serie de etiquetas con las que asignar a los migrantes. Los mensajes se fundamentan más en un juicio emocional que en cualquier tipo de argumento racional. Por tanto, cómo se denomine a los migrantes será fundamental para que se produzca finalmente la cohesión social.

\section{Referencias bibliográficas}

Acosta, T. (2015). Encuadres sobre inmigración reproducidos en YouTube. Universidad de Salamanca.

Alesina, A., Miano, A. \& Stefanie, S. (2018). Immigration and redistribution. (No. w24733). National Bureau of Economic Research.

Alonso, L. (2018). El discurso populista en Twitter. Un análisis comparado del estilo comunicativo de los actores políticos populistas de España, Italia, Francia y Reino Unido. Universitat Jaume I.

Bañón, A. (2002). Discurso e inmigración. Propuestas para el análisis de un debate social. Universidad de Murcia. 
Bar-Tal, D., Chernyak-Hai, L., Schori, N. \& Gundar, A. (2009). A sense of self-perceived collective victimhood in intractable conflicts. International Review of the Red Cross, Cambridge, 91(874), 229-258.

Bourdieu, P. (1977). Sur le pouvoir symbolique. Annales, mayo-junio, 405-411.

Cantó, P. (2018). Las mentiras que se repiten sobre los migrantes a bordo del 'Aquarius', en Verne. El País. https://verne.elpais.com/verne/2018/06/12/articulo/1528794173_525601.html

Cea D’Ancona, M. A. (2009). La sociedad española ante los nuevos modelos de familia. Panorama social, (10), 8-22.

Citron, D. \& Norton, H. (2011). Intermediaries and Hate Speech: Fostering Digital Citizenship for Our Information Age. Boston University Law Review, (91), 1435.

https://ssrn.com/abstract $=1764004$

Código penal y legislación complementaria. Códigos electrónicos (2019). BOE, Ministerio de Justicia, España. https://www.boe.es/legislacion/codigos/abrir_pdf.php?fich=038_Codigo_Penal_y_legislacion_co mplementaria.pdf

Comisión Europea contra el Racismo y la Intolerancia. Consejo de Europa (2015), en Recomendación General $n^{o} 15$ sobre Líneas de Actuación para combatir el discurso de odio y Memorándum explicativo.

http://www.mitramiss.gob.es/oberaxe/ficheros/documentos/2016_12_21Recomendacion_ECRI_NO_15_Discurso_odio-ES.pdf

Cortina, A. (2017). Aporofobia, el rechazo al pobre. Un desafío para la democracia. Paidós.

De la Fuente, M. (2006). La argumentación en el discurso periodístico sobre la inmigración. Universidad de León.

Gallardo, C. Ma . (2008). Migración y mujer: para un mundo mejor, en J. García Roca y J. Lacamba (Coords.), VV. AA., La inmigración en la sociedad española. Edicions Bella Terra.

Imbert, G. (1990). Los discursos del cambio. Imágenes e imaginarios sociales en la España de la Transición (1976-1982). Akal.

Lafuente, P. (2010). Tratamiento informativo de la inmigración extranjera en los principales diarios españoles. Comunicación y desarrollo en la era digital: Congreso AE-IC.

Malditobulo (2018a). Los bulos sobre los refugiados y migrantes del Aquarius. https://maldita.es/malditobulo/los-bulos-sobre-los-refugiados-y-migrantes-del-aquarius/

Malditobulo (2018b). La CIA ha avisado al Gobierno de que entre los migrantes del Aquarius hay soldados de Boko Haram" [imagen digital]. https://migracion.maldita.es/bulo/no-la-cia-no-haavisado-al-gobierno-de-que-entre-los-migrantes-del-aquarius-hay-soldados-de-boko-haram/

Mantzarlis, A. (2017). 18 Simposio Internacional de Periodismo Online (ISOJ), Austin, EE.UU., abril 21 [Vídeo]: https://www.youtube.com/watch?v=rtSY4DuEdBA\&feature=youtu.be 
Olmo y Romero, J. A. (2019). Desinformación: Concepto y perspectivas. Análisis del Real Instituto Elcano (ARI), (41).

http://www.realinstitutoelcano.org/wps/portal/rielcano_es/contenido?WCM_GLOBAL_CONTEX T=/elcano/elcano_es/zonas_es/ari41-2019-olmoromero-desinformacion-concepto-y-perspectivas

Peirano, M. (2019). El enemigo conoce el sistema: manipulación de ideas, personas e influencias después de la economía de la atención. Debate sociedad.

Retis, J. (2003). La construcción de la imagen de la inmigración latinoamericana en la prensa española, en Comunicación, cultura y migración. Dirección General de Políticas Migratorias.

Revilla, A. (2011). Una buena praxis en el tratamiento informativo de la inmigración en tiempos de crisis, en La ética de la comunicación a comienzos del siglo XXI. Actas I Congreso Internacional de Ética de la Comunicación. Sevilla, España: Eduforma.

Richter, U. (2018). El ciudadano digital: Fake news y posverdad en la era de internet. Océano.

Santamaría, E. (2002). La incógnita del extraño. Una aproximación a la significación sociológica de la "inmigración no comunitaria. Anthropos.

Silverman, C. (2013). Verification handbook. http://verificationhandbook.com/additionalmaterial/

Van Dijk, T. (1997). Racismo y análisis crítico de los medios. Paidós.

Van Dijk, T. (2011). Sociedad y discurso. Cómo influyen los contextos sociales sobre el texto y la conversación. Gedisa Editorial.

\section{AUTORES:}

\section{Ángel Fernández Fernández}

Doctor en Comunicación por la Universidad Europea de Madrid (UEM), en la que defendió la tesis doctoral titulada Relatos híbridos: El papel de la narratividad en la visualización de información interactiva obteniendo la calificación de sobresaliente cum laude. Director del Máster Universitario en Comunicación y Emprendimiento Digital de la Universidad Europea. Su línea de investigación gira alrededor del estudio de la interactividad, las narrativas digitales basadas en datos, y el impacto cultural de las nuevas tecnologías. Es miembro del Grupo de investigación Comunicación y Migraciones e investigador en el grupo UE-Medios del proyecto INMIGRA3-CM.

angelmiguel.fernandez@universidadeuropea.es

Orcid ID: https://orcid.org/0000-0003-4264-7788

Google Scholar: https://scholar.google.com/citations?user=fUIAWAsAAAAJ\&hl=en\&oi=sra

\section{Almudena Revilla Guijarro}

Doctora en Ciencias de la Información por la Universidad Complutense de Madrid. Profesora titular en Creación Literaria y Comunicación en la Universidad Europea de Madrid. Actualmente es investigadora en el grupo UE-Medios del proyecto INMIGRA3-CM y en el proyecto interno UE Monitorización y análisis de la caracterización discursiva de los relatos migratorios en Twitter. Miembro fundador del Grupo de investigación DILE (Discurso y Lengua Española) de la UAM. almudena.revilla@universidadeuropea.es

Orcid ID: https://orcid.org/0000-0002-1021-2678

Google Scholar: https://scholar.google.com/citations?user=eGqwbCwAAAAJ\&hl=es 


\section{Lucía Andaluz Antón}

Doctoranda en la Universidad Europea de Madrid (UEM) bajo la temática "Estudio del modelo de fact-checking y su aplicación en Twitter, a través del caso de estudio sobre migración en el Aquarius: Implicación de la carga emocional en la difusión de los mensajes, como estrategia para la viralización de Desinformación". Su línea de investigación gira en torno al estudio de las nuevas iniciativas y técnicas de fact-checking, así como de la desinformación en red. Actualmente es investigadora en el proyecto de investigación "Monitorización y análisis de la caracterización discursiva de los relatos migratorios en Twitter (2018/UEM07)". Coordinadora de Prácticas de Postgrado en la Facultad de CC. Sociales y de la Comunicación en la Universidad Europea de Madrid.

marialucia.andaluz@universidadeuropea.es

Orcid ID: https://orcid.org/0000-0001-8540-8328 